\title{
Evidence for biodegradation products in the interstellar medium
}

\author{
Kani Rauf and Chandra Wickramasinghe \\ Cardiff Centre for Astrobiology, Cardiff University, 2 North Road, Cardiff CF10 3DY, UK \\ e-mail:Wickramasinghe@cf.ac.uk
}

\begin{abstract}
The interstellar absorption band centred on $2175 \AA$ that is conventionally attributed to monodisperse graphite spheres of radii $0.02 \mu \mathrm{m}$ is more plausibly explained as arising from biologically derived aromatic molecules. On the basis of panspermia models, interstellar dust includes a substantial fraction of biomaterial in various stages of degradation. We have modeled such an ensemble of degraded biomaterial with laboratory spectroscopy of algae, grass pigments, bituminous coal and anthracite. The average ulrtraviolet absorption profile for these materials is centred at $2175 \AA$ with a full width at half maximum of $250 \AA$, in precise agreement with the interstellar extinction observations. Mid-infrared spectra also display general concordance with the unidentified interstellar absorption features found in a wide variety of astronomoical sources.
\end{abstract}

Received 27 September 2009, accepted 18 October 2009, first published online 20 November 2009

Key words: degradation products of biomateria, interstellar dust, panspermia, polycyclic aromatic hydrocarbon, interstellar molecules.

\section{Introduction}

Some four decades ago, ultraviolet (UV) stellar spectroscopy was born with the deployment of telescopes and instruments flown above the atmosphere. When stellar spectra were first examined at wavelengths shortward of $3000 \AA$, an interstellar absorption band centred at $2175 \AA$ with a half-width at full maximum of $\sim 250 \AA$ was discovered in the spectra of most reddened stars (Stecher 1965; Wickramasinghe 1967). The average interstellar extinction curve showing this feature is shown in Fig. 1, where the observed interstellar extinction curve is decomposed theoretically into three components. Component 1 represents scattering by dielectric grains with sizes and properties resembling bacteria, Component 2 is the extinction due to an absorber producing the mid-UV band, and component 3 represents a population of nanometricsized dielectric particles (Hoyle \& Wickramasinghe 1999; Wickramasinghe et al. 2009).

Whilst the mean extinction curve for dust in the solar vicinity is well represented by the uppermost curve in Fig. 1, stars in other regions of the Galaxy and in external galaxies show a modest degree of variability. Such variability is consistent with differing proportions of the components 1, 2 and 3. Fig. 2, from Boulanger et al. (1994), shows how individual stars show varying relative amounts of the $2175 \AA$ carrier. Boulanger et al. (1994) also discuss the correlation between the strength of the UV absorption peak and the mid-infrared (IR) emission bands that could be interpreted as arising from a common molecular carrier (Hoyle \& Wickramasinghe 1989).
The attribution of the $2175 \AA$ extinction bump by Hoyle and Wickramasinghe to small graphite spheres is now widely accepted, with such a component included in most models of interstellar grains (Hoyle \& Wickramasinghe 1962, 1969; Mathis 1990; Draine 2003). There are, however, serious problems associated with the graphite model (Wickramasinghe et al. 1992). The requirement of graphite, which has a planar lattice structure, to be in the form of small spheres in interstellar space appears scarcely plausible, as indeed does the requirement that the size dispersion around a central size $0.02 \mu \mathrm{m}$ be vanishingly small. Alternative molecular explanations of the $2175 \AA$ absorption, first proposed by Hoyle and Wickramasinghe in 1977, involve aromatic organic molecules (Hoyle \& Wickramasinghe 1977). In this paper we show that such molecules are most reasonably interpreted as being degradation products of biology.

\section{Microbial dust and polycyclic aromatic hydrocarbons}

Some recent models of interstellar dust have included an abiotic polycyclic aromatic hydrocarbon (PAH) component that, under appropriate circumstances, could produce a $\lambda=$ $2175 \AA$ absorption band (Witt et al. 2006). However, for PAHs and other complex organic molecules to form abiotically poses serious problems, in particular with the requirement that compact PAHs are in high ionization states. An alternative model that fits the general context of panspermia is that organic dust and molecules in interstellar space include a substantial component derived from biology itself. 


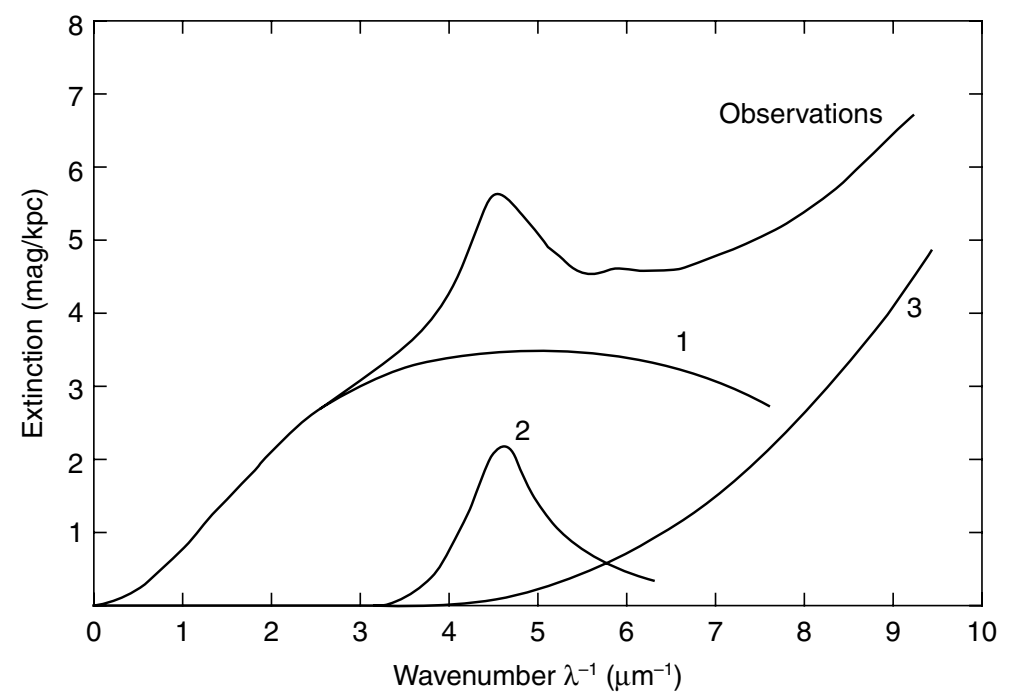

Fig. 1. Interstellar extinction observations from a compilation from Sapar \& Kuusik (1978) interpreted as a composite of (1) dielectric grains of radii $0.3 \mu \mathrm{m}$, (2) molecular absorption due to aromatics and (3) dielectric particles of radii $0.01 \mu \mathrm{m}$.

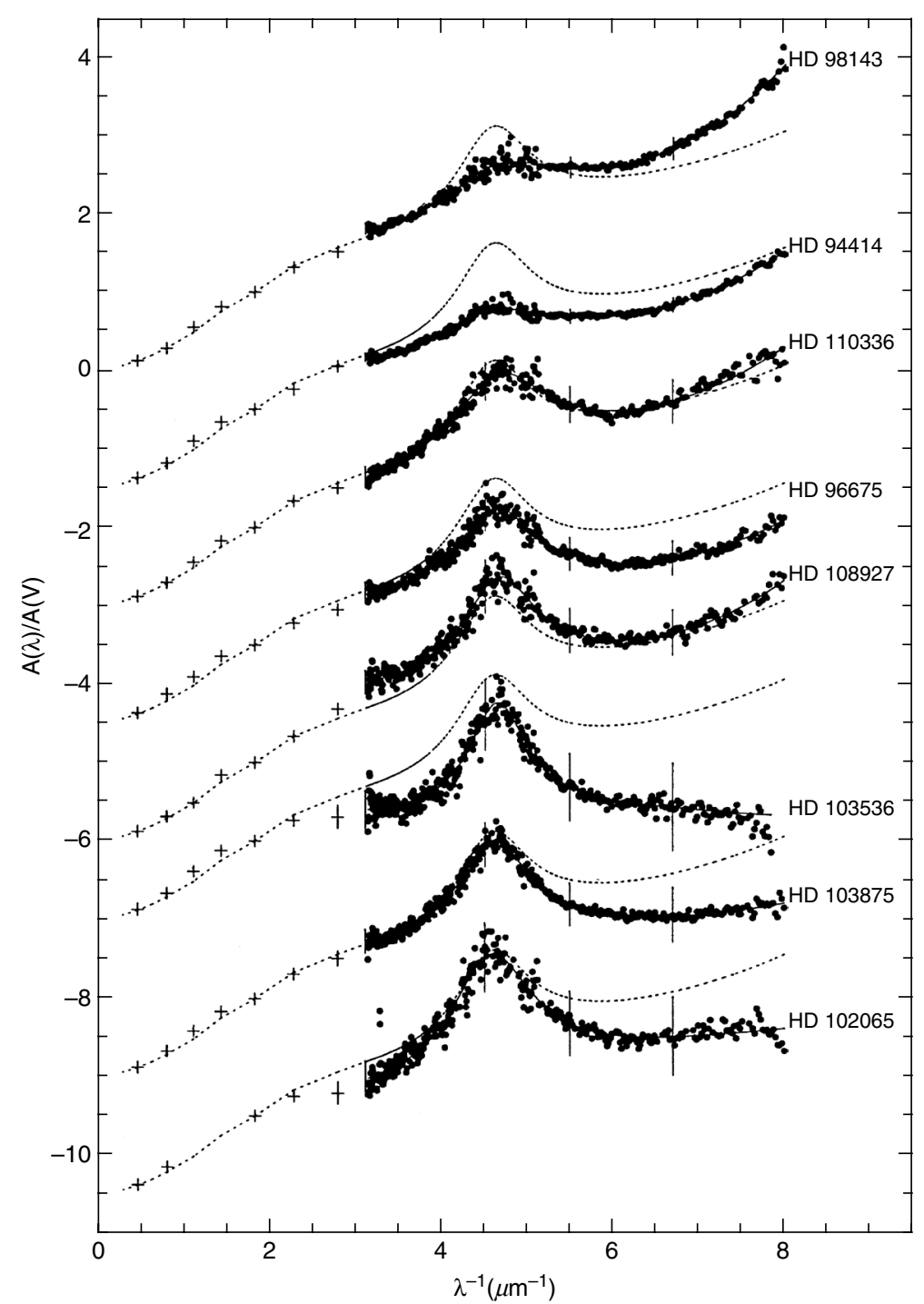

Fig. 2. Normalized interstellar extinction curves for stars in our Galaxy showing varying strengths of the $2175 \AA$ absorption feature adapted from Boulanger et al. (1994). Crosses and the dashed curve represent mean interstellar extinction. 


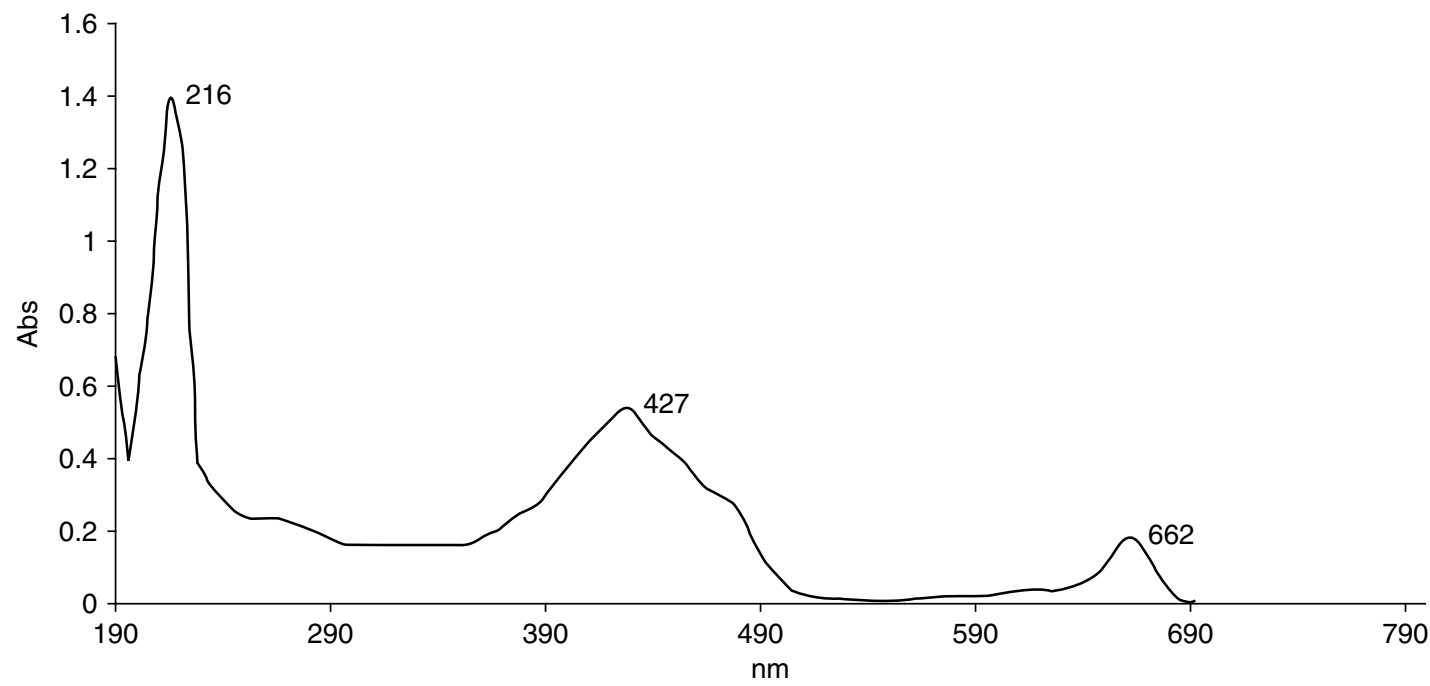

Fig. 3. UV-visible spectrum of algae dissolved in cyclohexane.

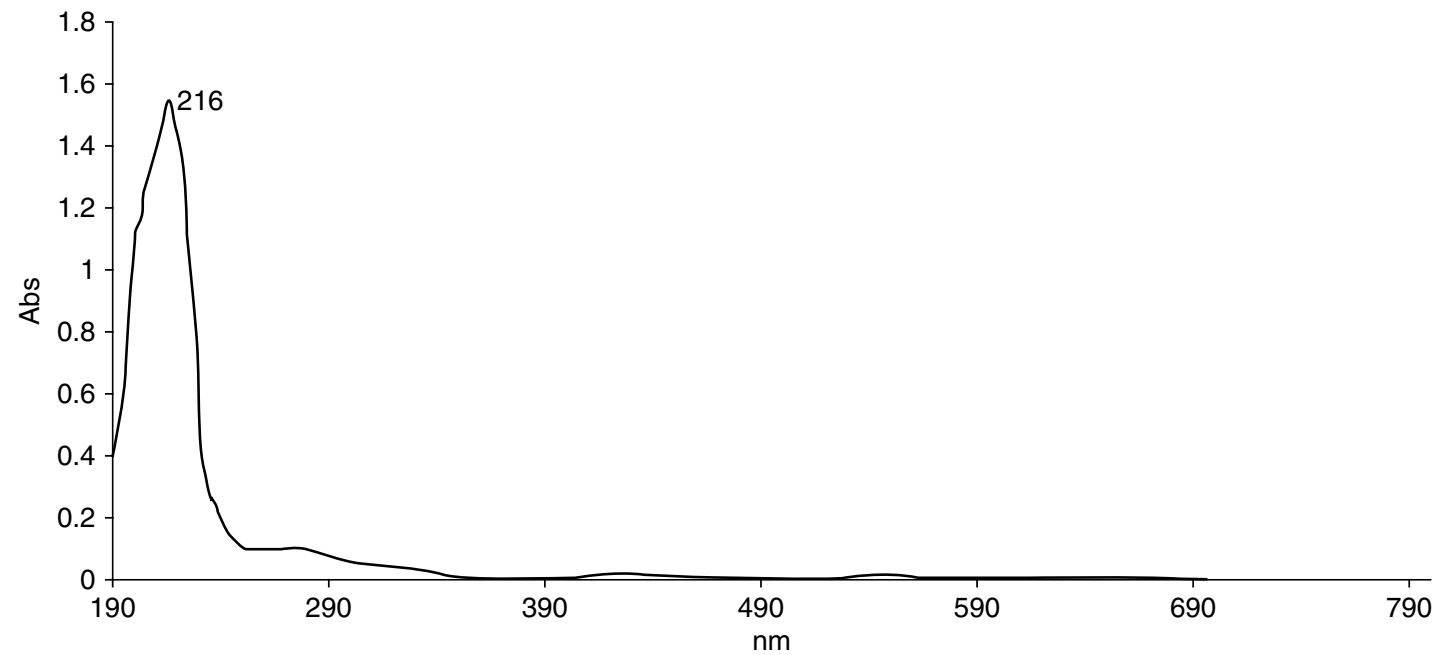

Fig. 4. UV-visible spectrum of grasses dissolved in cyclohexane.

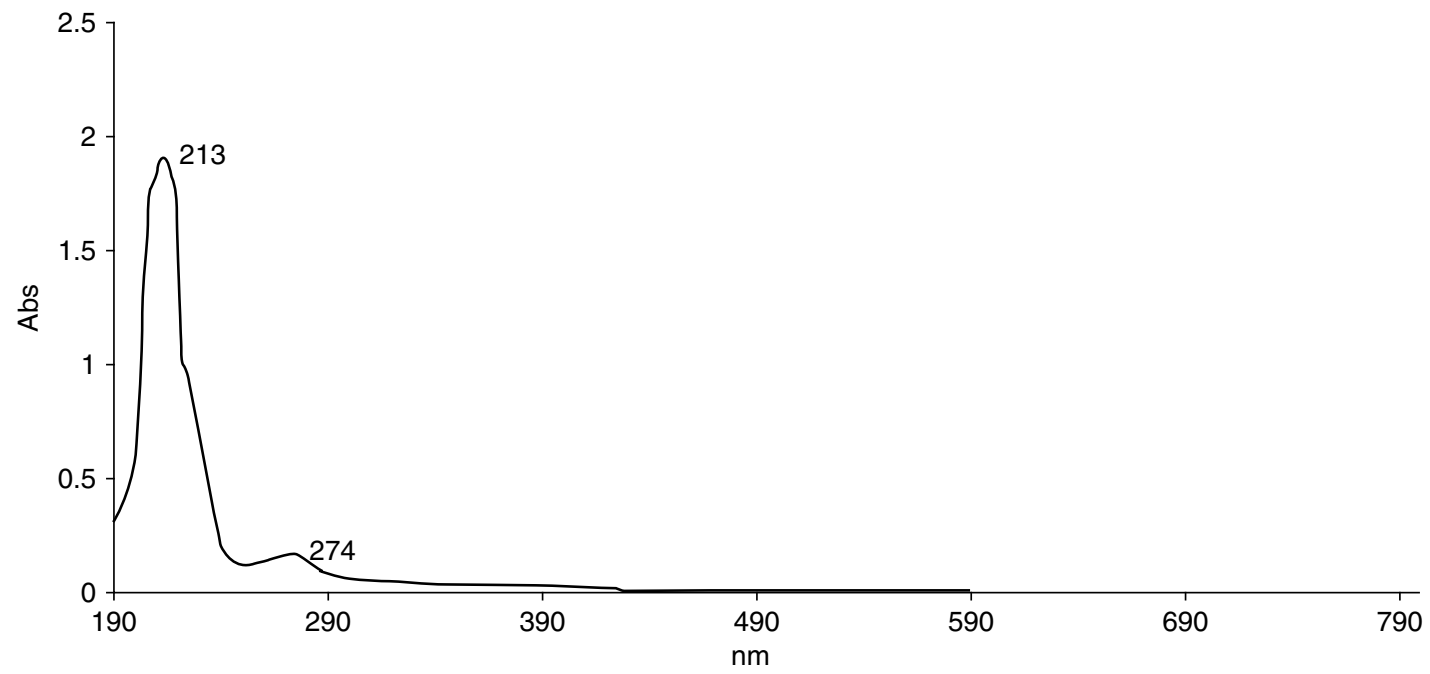

Fig. 5. UV-visible spectrum of anthracite dissolved in cyclohexane. 


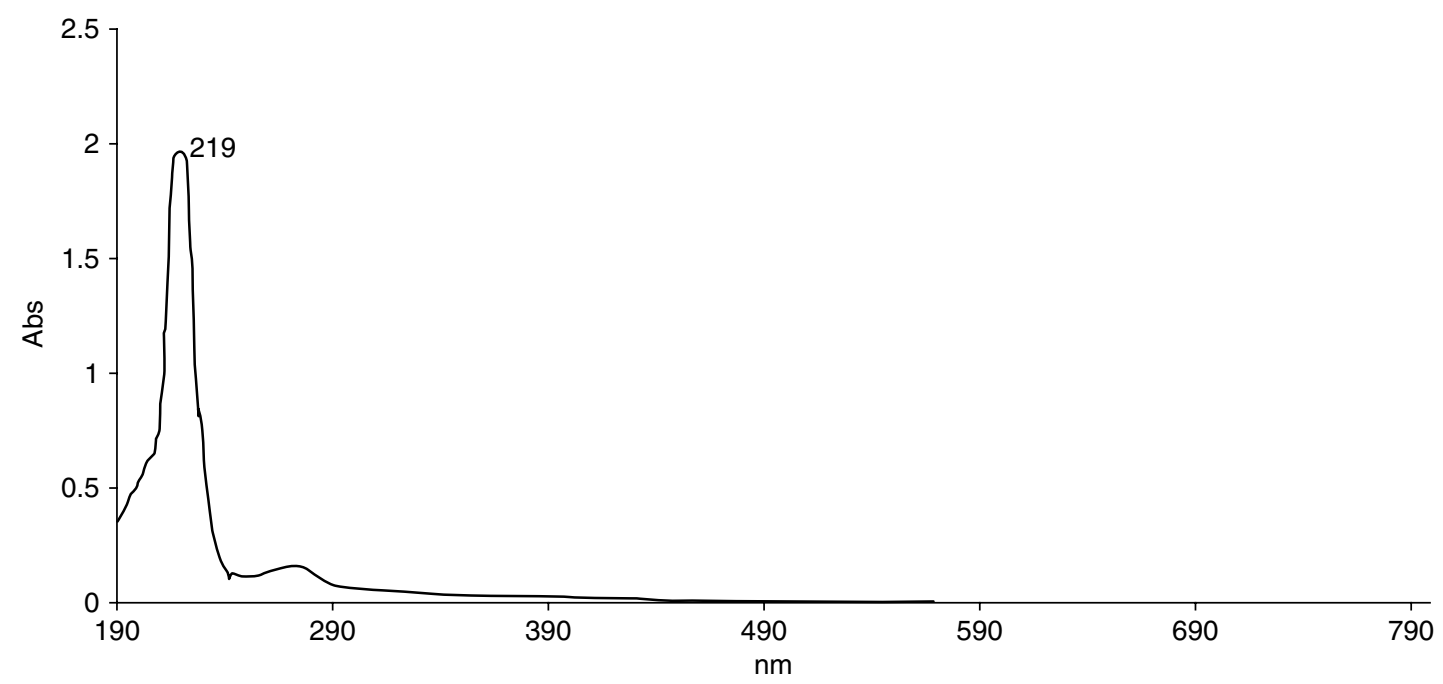

Fig. 6. UV-visible spectrum of semi-anthracite dissolved in cyclohexane.

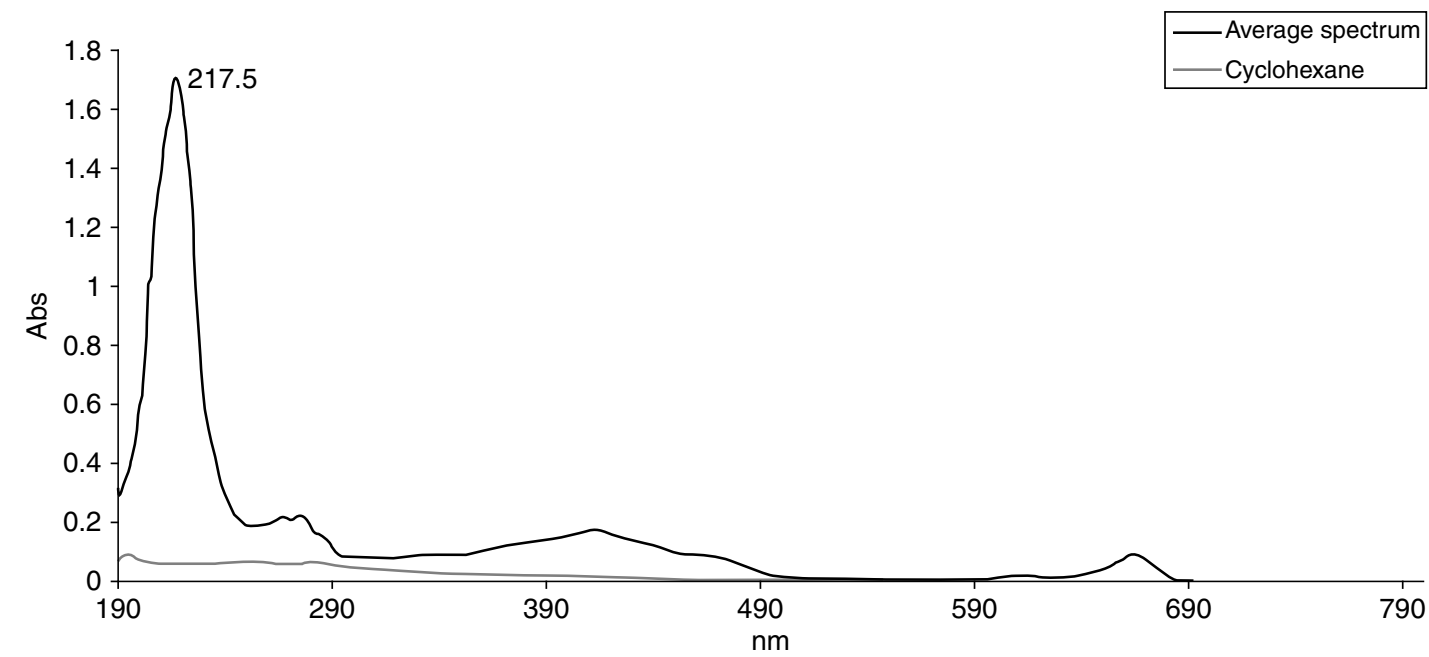

Fig. 7. Average spectrum of a putative interstellar microbiota in various stages of degradation.

According to this point of view, microbial cells replicate in liquid interiors of primordial comets and are distributed widely across the galaxy (Wickramasinghe et al. 2009). Interstellar material would then include the detritus of biology microbial cells in various stages of degradation and break-up. The degradation of cells in HII regions and in other high radiation environments is of course inevitable. Whilst wholesale destruction, if it occurs, would vitiate a panspermia model, the requirement for panspermia to operate unhindered is that a fraction, less than one part in $10^{20}$, survives from one amplification site to the next; this condition would seem impossible to violate.

\section{Experiments and materials used}

Our model thus leads to a component of interstellar dust grains that start off as intact viable cells and end up as coal-like grains. In order to model the effects of sequential degradation we took algal cells and grasses as a starting point, and measured the absorption properties of the following:

(1) algae and grasses;

(2) semi-anthracite;

(3) anthracite.

Samples (2) and (3) represent the last stages in a putative biodegradation sequence.

The sources of the material were as follows:

- Oedogonium sp (a fresh water species of green algae) collected from Roath Park, Cardiff;

- grass from the Santa Cruz island of the Galapagos provisionally identified as Panicum maximum;

- both bituminous coal (semi-anthracite) and anthracite were obtained from Geo Supplies Ltd., UK.

We were primarily concerned with the precise placement of a $2175 \AA$ absorption feature in all of these systems, which were dispersed and dissolved in a non-polar solvent. We also 


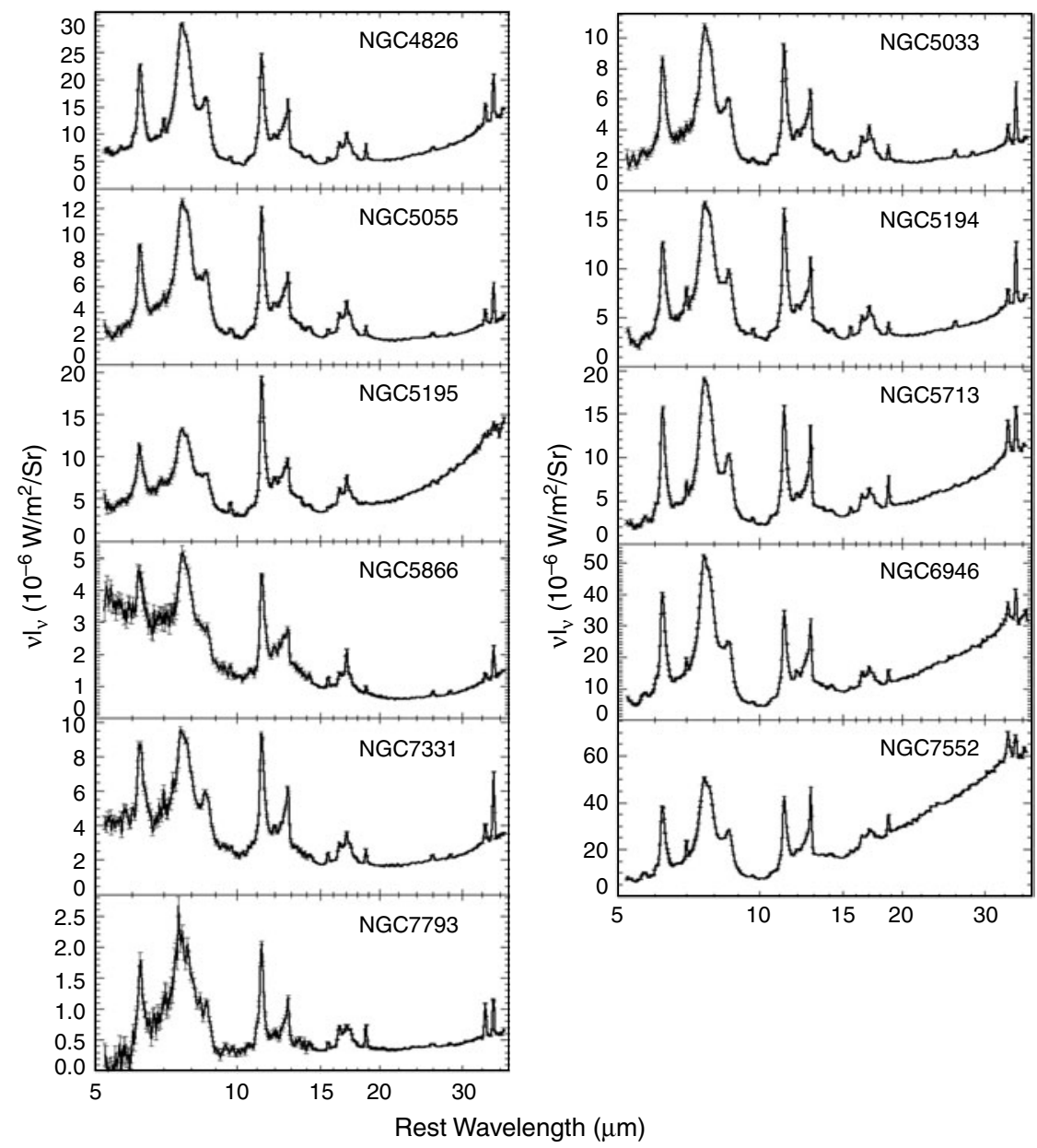

Fig. 8. Recent compilation of Spitzer telescope emission spectra of a variety of galactic sources showing PAH emissions (Smith et al. 2007).

examined mid-IR spectra of the same systems dispersed in $\mathrm{KBr}$ discs, with a view to comparing them with the Unidentified IR Bands (UIBs) in interstellar material.

\section{Methods of sample mounting and preparation}

For UV and visual spectroscopy, the samples were dissolved in cyclohexane - a non-polar solvent. Algae, grasses, semi-anthracite and anthracite (approximately $70 \mathrm{mg}$ in each case) were individually added to cyclohexane $(500 \mu l)$ and dissolved. The use of a non-polar solvent eliminates the effect of wavelength shifts. All samples were agitated for a few minutes and allowed to stand still on the bench for 15 minutes to allow the sedimentation to complete. A clear solution $(100 \mu \mathrm{l})$ was carefully pipetted out from the top and placed in a test tube containing the solvents $(3.5 \mathrm{ml})$. The tube was briefly agitated and transferred into a spectrophotometer (JASCO V-570 UV/visible imaging system (VIS)/near infrared (NIR) spectrophotometer).

For IR spectroscopy, the samples were first subjected to vigorous grinding using an agate pestle until a fine powder was obtained. The sample $(0.2 \mathrm{mg})$ was embedded in $\mathrm{KBr}$ $(20 \mathrm{mg})$, and the resulting mixture was transferred into a small blending mill $\left(\right.$ SPECAmill ${ }^{\mathrm{TM}}$, Kent, UK) for further grinding. After 10 minutes, the sample was subjected to
Table 1. Distribution of two astronomical observations (UIBs and proto-planetary nebulae (PPNe)) and major IR absorption bands in laboratory models of terrestrial origin.

\begin{tabular}{lccccc}
\hline UIBs & PPNe & Algae & Grasses & $\begin{array}{l}\text { Bituminous } \\
\text { coal }\end{array}$ & $\begin{array}{l}\text { Anthracite } \\
\text { coal }\end{array}$ \\
\hline 3.3 & 3.3 & 3.3 & - & 3.3 & 3.3 \\
- & 3.4 & 3.4 & 3.4 & 3.4 & 3.4 \\
6.2 & 6.2 & 6.0 & 6.1 & 6.2 & 6.2 \\
- & 6.9 & 6.9 & 6.9 & 6.9 & 6.9 \\
- & 7.2 & 7.2 & 7.2 & 7.2 & 7.2 \\
7.7 & 7.7 & - & 7.6 & - & 7.7 \\
- & 8.0 & 8.0 & 8.0 & - & - \\
8.6 & 8.6 & 8.6 & - & - & - \\
11.3 & 11.3 & 11.3 & 11.1 & 11.5 & 11.3 \\
- & 12.2 & 12.1 & 12.05 & 12.3 & 12.5 \\
- & 13.3 & - & - & - & 13.4 \\
\hline
\end{tabular}

drying under high vacuum for 3-4 hours, after which it was subsequently transferred into the Evacuable Pellet Dies (SPECAC Ltd, Kent, UK) to make the $\mathrm{KBr}$ disc. The resulting $\mathrm{KBr}$ disc was analysed using a Fourier transform IR (FTIR) spectrometer (JASCO Model FT/IR 660 Plus series). 


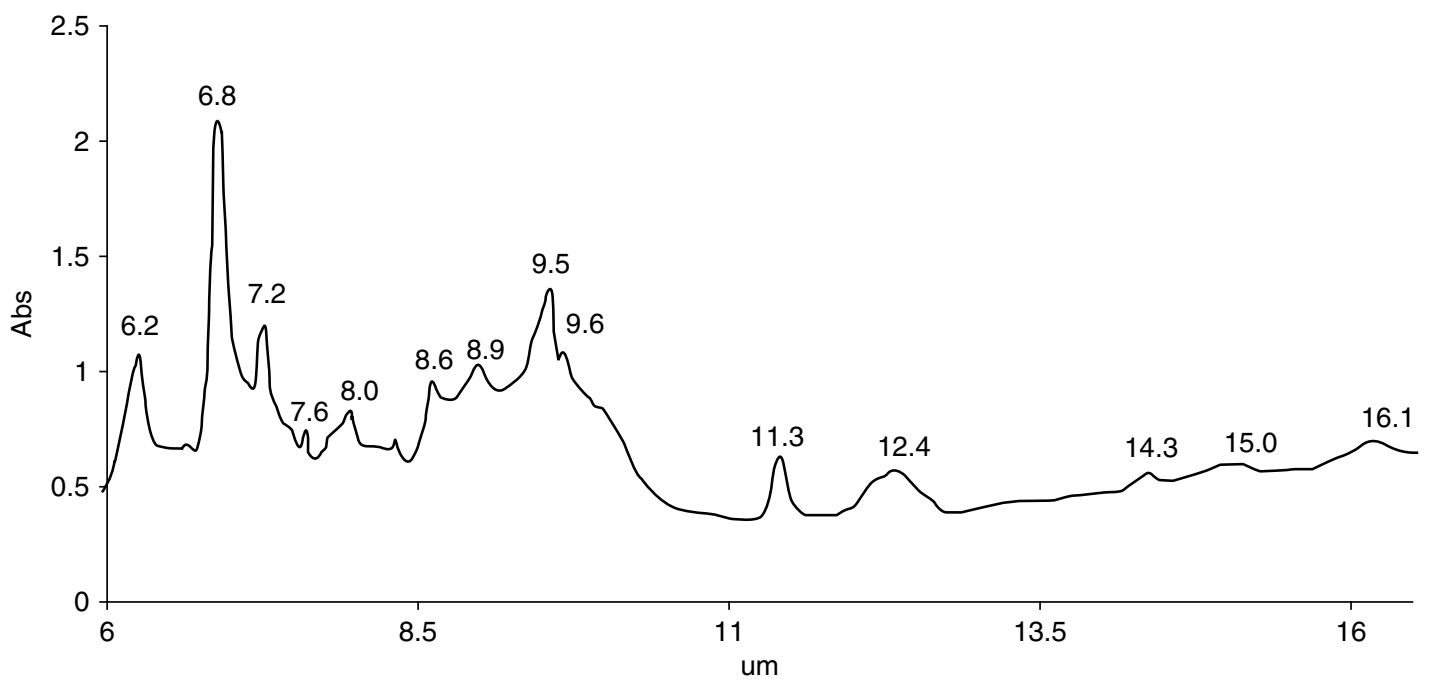

Fig. 9. Spectrum of algae dispersed in $\mathrm{KBr}$ disc.

\section{Results and conclusion}

\section{Ultraviolet}

Figures 3-6 show UV spectra of algae, grasses, semi-anthracite and anthracite, representing the UV-visual spectra of a putative interstellar microbiota in various stages of degradation. Figure 7 shows the average absorption spectrum of the whole sequence, a normalization being carried out to give an optical depth of 1.7 at the peak. We are particularly impressed with the precise correspondence between the $2175 \AA$ peak in Fig. 7 and the interstellar extinction observations (Figs 1 and 2). The full width at half maximum (FWHM) of $250 \AA$ agrees almost precisely with that of the interstellar absorption feature. Earlier attempts to construct an average UV spectrum from 115 aromatic biomolecules led to a synthetic $2175 \AA$ band that is in general agreement with the curve in Fig. 7 (Wickramasinghe et al. 1989).

\section{Infrared}

Mid-IR spectra for a large number of galactic and extragalactic sources have recently been obtained using the Spitzer Space Telescope (Smith et al. 2007). A typical selection of such spectra are shown in Fig. 8.

The detailed spectra are variable from source to source, but strong recurring peaks occur at the wavelengths listed in Table 1. The IR spectra of the materials we studied also show peaks at wavelengths in general correspondence with the astronomical peaks. It has been suggested by many authors that these peaks arise from PAH structures, but objections cited initially by Schlemmer et al. (1994) would still appear to be largely valid. Moreover the formation of PAHs abiotically also poses problems. Our laboratory spectra exhibited absorptions at most of the relevant astronomical wavelengths as is seen in Table 1 .

Figure 9 shows the IR spectrum of our sample of algae, which is seen to correspond approximately with portions of the spectra displayed in Fig. 8. It should be noted, however, that whilst Fig. 7 shows emission spectra arising from molecular fragments (detritus of biomaterial?) under various excitation conditions, Fig. 9 shows the absorption spectrum of molecules within biological particles crushed and fused into $\mathrm{KBr}$ disks. Traces of water cannot be ruled out in the sample, with a strong 2.9-3 $\mu \mathrm{m}$ band (not shown in Fig. 9) that is almost certainly attributed to $\mathrm{H}_{2} \mathrm{O}$, whilst the elevated complex of features around $10 \mu \mathrm{m}$ is almost certainly assignable to polysaccharides.

In conclusion we note that the interstellar absorption feature at $2175 \AA$ in stellar spectra is plausibly attributed to aromatic molecules within biological cells. The unidentified interstellar absorption bands in the IR spectra may also have an origin in the same biomolecular structures (Hoyle \& Wickramasinghe 1989).

\section{References}

Boulanger, R., Prevot, M.L. \& Gry, C. (1994). Astron. Astrophys. 284, 956. Draine, B.T. (2003). Ann. Rev. Astron. Astrophys. 41, 241.

Hoyle, F. \& Wickramasinghe, N.C. (1962). Mon. Not. Roy. Astron. Soc. 124, 417.

Hoyle, F. \& Wickramasinghe, N.C. (1969). Nature 223, 459.

Hoyle, F. \& Wickramasinghe, N.C. (1977). Nature 270, 323.

Hoyle, F. \& Wickramasinghe, N.C. (1989). ESA SP 290, 67.

Hoyle, F. \& Wickramasinghe, N.C. (1999). Astronomical Origins of Life. Kluwer Academic Publishers, Dordrecht.

Mathis, J.S. (1990). Ann. Rev. Astron. Astrophys. 28, 37.

Sapar, A. \& Kuusik, I. (1978). Publ. Tartu. Astrophys. Obs. 46, 71.

Schlemmar, S. et al. (1994). Science 265, 1685.

Smith, J.D.T. et al. (2007). Astrophys. J. 656, 770

Stecher, T.P. (1965). Astrophys. J. 142, 1683.

Wickramasinghe, J.T., Wickramasinghe, N.C. \& Napier, W.M. (2009). Comets and the Origin of Life. World Scientific Publ. Co., Singapore.

Wickramasinghe, N.C. (1967). Interstellar Grains. Chapman \& Hall, London.

Wickramasinghe, N.C., Hoyle, F. \& Al-Jabory, T. (1989). Astrophys. Space Sci. 158, 135.

Wickramasinghe, N.C., Wickramasinghe, A.N. \& Hoyle, F. (1992). Astrophys. Space Sci. 196, 167.

Witt, A.N. et al. (2006). Astrophys. J. 636, 303. 